\title{
Mathematical model on surface reaction diffusion in the presence of front chemical reaction
}

\author{
D.V. Permikin, V.S. Zverev* \\ Department of Mathematical Physics, Urals Federal University, Lenin Ave. 51, Ekaterinburg 620083, Russian Federation
}

\section{A R T I C L E I N F O}

\section{Article history:}

Received 15 May 2012

Received in revised form 3 August 2012

Accepted 8 October 2012

Available online 6 November 2012

\section{Keywords:}

Surface reaction diffusion

Unknown moving boundary

System of nonlinear parabolic equations

Asymptotic and numeric solution

\begin{abstract}
A B S T R A C T
The article discusses a mathematical model of solid-phase diffusion over substance surface accompanied a frontal chemical reaction. The purpose of our article is to describe the concentration distribution and surface reacted layer growth. The model is a system parabolic equations, complicated with the presence of mobile front. It takes account of diffusive fluxes redistribution, sublimation from the surface, chemical reaction reversibility. The asymptotic approximation of the obtained nonlinear problem is constructed. Numerical solution was also carried out. Both numerical and analytical solutions conform to each other in a wide range of parameter changes, whereas observed differences are explained. It was obtained that the reaction front at the substrate surface grows as the fourth root of time in the assumed absence of evaporation and reaction reversibility. In the presence of evaporation the logarithmic distribution law $\ln (t)$ is obtained. The theoretical possibility of sharp deceleration and stop of reaction product layer growth is obtained.
\end{abstract}

(c) 2012 Elsevier Ltd. All rights reserved.

\section{Introduction}

In the course of the last decades film technologies have been widely used in microelectronics; they were in the basis of integrated optics elements production, vacuum obtaining and artificial corrosion-resistant coating production, etc. There is a large number of film obtaining techniques [1].

During investigation of the method for creating thin films it was discovered the phenomenon of rapid solid-phase spreading couple with a chemical reaction. Experimental research is carried out by by the method of "contact diffusion annealing" [2]. Reagents were shaped as disks, and the diffusant is more less then the substrate (Fig. 1). The main quantitative characteristic of the process is surface layer length $l_{s}$. It was found that "spreading of the diffusant on the substrate surface is retarded sharply" [2], when a certain critical value is achieved. Visible surface reaction interface almost stops (Fig. 2), and this is not typical for the diffusion processes. The phenomenon was termed surface-reaction diffusion (SRD).

Experimental investigations have shown that diffusion over the substrate surface passes rapidly, whereas reaction front distribution inside- slowly. The effect actively appears at solid-phase interaction of the following substances: $\mathrm{CoO}$ and $\mathrm{WO}_{3} ; \mathrm{CuO}$ and $\mathrm{MoO}_{3}$; $\mathrm{ZnO}$ and $\mathrm{WO}_{3} ; \mathrm{Pb}_{2} \mathrm{MoO}_{5}$ and $\mathrm{MoO}_{3}$ [2,3]. There are two preconditions for surface-reaction diffusion: a low surface energy and a high surface mobility of the diffusant.

\footnotetext{
* Corresponding author. Tel.: +7 3433507541.

E-mail address: v-s-zverev@yandex.ru (V.S. Zverev).
}

There are some similarities between mass transfer in surfacereaction diffusion and the model of diffusion in polycrystalline materials, where surface diffusion is rapid and diffusant outflows to the granules. It is the classic subdiffusion Fisher's model - "diffusion in bicrystal" [4] and its analogues - Suzuoka and Whipple models [5-7]. However these models don not explain the growth deceleration of the surface layer length.

In our case the process of surface-reaction diffusion is complicated by mobile reaction front, chemical reaction reversibility, diffusing substance evaporation from the open substrate surface (Fig. 1). Such systems are found in other technologies of material production (see, among others, [8-11]), geophysics [12-14] and medical physics [15]. A reversible chemical reaction and a processes of evaporation have been studied by various research ([16-18]).

The mobile reaction front leads up to the necessity to consider the of partial derivatives equations with unknown moving boundary. The classic problem with moving boundary is the Stefan problem. Other examples can be the following problems: solidification-meltdown [19], wound healing [20], concrete carbonation $[21,22]$, issues concerning processes of evaporation, condensation $[17,23]$. There are not general solution techniques for problems with unknown moving boundaries, and virtually any of such nonlinear problems requires developing special approaches. Sufficiently detailed survey of Stefan-like problem and its applications is described in works [24-27].

In this article we analyze a mathematical model of SRD under condition of an "inexhaustible source", diffusion fluxes redistribution, chemical reaction reversibility and diffusant evaporation. 


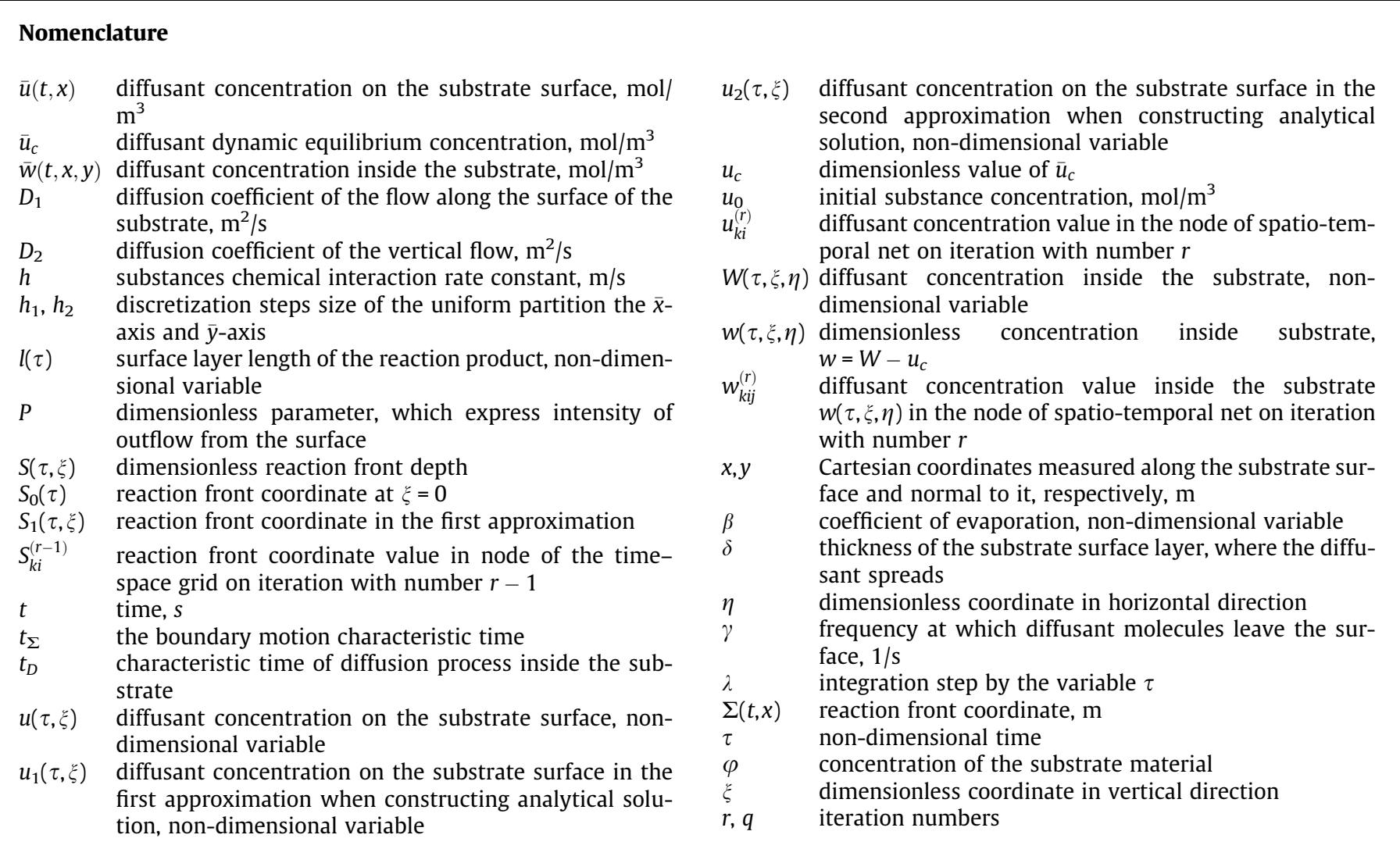

The possibility of sharp deceleration of the surface substrate layer growth is obtained.

In Section 2 the model of surface-reaction diffusion is described. We present analytical and numerical solutions in Sections 3 and 4, accordingly. In the final paragraph we discusses results and compares models of surface-reaction diffusion (model without reaction reversibility and without evaporation, model with evaporation, model with reaction reversibility and generalized model).

\section{The model}

Generalized model construction based on the following assumptions.

1. Coefficient of diffusion over the substrate surface $D_{1}$ is much greater than that of its volume $D_{2}$. This makes possible to divide complete diffusive flux into two interconnected fluxes: diffusant flux over the substrate surface and flux from the surface layer inside the substrate.

2. The diffusant spreads over the surface in thin surface layer with thickness $\delta$, whereas reaction proceeds inside the substrate.

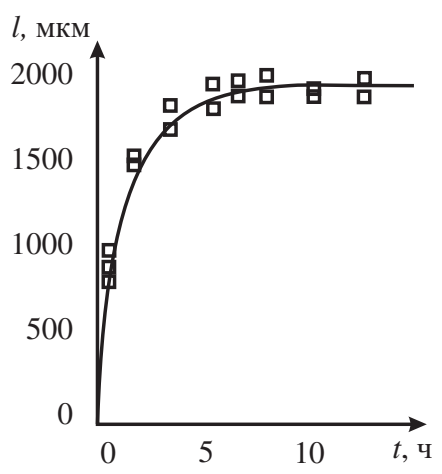

Fig. 2. Surface-reaction diffusion experimental investigation in the $\mathrm{CoO}-\mathrm{WO}_{3}$ system at temperature $900{ }^{\circ} \mathrm{C}[2]\left(l_{s}-\right.$ surface layer length of the reaction product).

3. Chemical reaction rate constant is quite high, and it makes possible to concentrate reactionary interaction on some reaction front (moving boundary). There is a first-order chemical reaction between oxides.

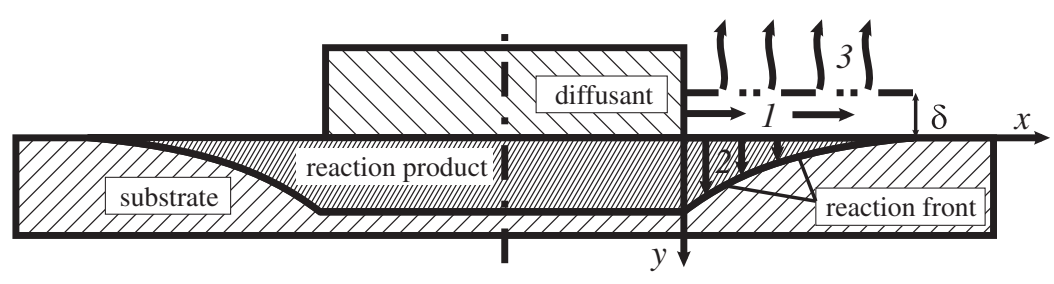

Fig. 1. Diffusion fluxes division diagram. Figure indicate the diffusant flows. 
4. The reaction is reversible and there is a concentration of diffusant dynamic equilibrium $\bar{u}_{c}$. This means that chemical reaction front is moving forward, when diffusant concentration is over threshold value $\bar{w}(t, x, y)>\bar{u}_{c}$.

5. Since diffusant spreads in thin surface layer with thickness $\delta$, then there is evaporation out of this layer from the whole surface of the substrate.

6. For analysis purposes we will not take into account that the substrate and the diffusant source have cylindrical form. The problem considered below is in Cartesian coordinate system, Fig. 1.

7. Problem formulation is isothermal; it does not take account of temperature field as a result of evaporation and reaction progress. Such simplification takes place because experimental research was held for hours in thermostat ovens at constant temperature, and temperature field flattening occurs much faster than that of diffusion and on "diffusive" time scales the temperature field can be considered homogenous.

Thus, diffusant concentration on the substrate surface $\bar{u}(t, x)$ is changed as a result of the substance transfer over the surface, outflow inside the substrate and evaporation.

$\frac{\partial \bar{u}}{\partial t}=D_{1} \frac{\partial^{2} \bar{u}}{\partial x^{2}}+\left.\frac{D_{2}}{\delta} \frac{\partial \bar{w}}{\partial y}\right|_{y=0}-\gamma \bar{u}, \quad x>0, \quad t>0$.

At the initial time there is no diffusant in the substrate, neither on its surface

$\bar{u}(0, x)=0, \quad \bar{u}(t, 0)=u_{0}, \quad \bar{u}(t, \infty)=0$.

Penetrating into the substrate the substance enters into a chemical reaction and reaction product is formed in area $0<y<\Sigma$. The diffusant flux along the $O x$ axis is negligible, therefore the concentration inside the substrate is described by simple diffusion equation

$\frac{\partial \bar{w}(t, x, y)}{\partial t}=D_{2} \frac{\partial^{2} \bar{w}(t, x, y)}{\partial y^{2}}, \quad t>0,0<y<\Sigma$.

With zero initial condition and condition of concentration continuity on boundary

$\bar{w}(0, x, y)=0, \quad \bar{w}(t, x, 0)=\bar{u}(t, x)$.

On the free boundary $\Sigma(t, x)$ between oxides there is a first-order chemical, therefore one can write down second boundary condition for task (3) and equation of the boundary motion

$$
\begin{aligned}
& -\left.D_{2} \frac{\partial \bar{w}}{\partial y}\right|_{y=\Sigma}=\left.h H\left(\bar{w}-\bar{u}_{c}\right)\left(\bar{w}-\bar{u}_{c}\right)\right|_{y=\Sigma}, \\
& \frac{\partial \Sigma}{\partial t}=\left.\frac{h H\left(\bar{w}-\bar{u}_{c}\right)\left(\bar{w}-\bar{u}_{c}\right)}{\varphi}\right|_{y=\Sigma}, \quad \Sigma(0, x)=0,
\end{aligned}
$$

where $H\left(\bar{w}-\bar{u}_{c}\right)$ - Heaviside function

$H(z)= \begin{cases}1, & z>0, \\ 0, & z \leqslant 0 .\end{cases}$

The system of parabolic equations, boundary and initial conditions (1)-(6) describes generalized surface diffusion with reversible reaction occurring on the front and substance evaporation.

For further analysis it is convenient to introduce dimensionless parameters

$\tau=\frac{h^{2}}{D_{1}} t, \quad \xi=\frac{h}{D_{1}} x, \quad \eta=\frac{h}{D_{1}} y$,

$u(\tau, \xi)=\frac{\bar{u}}{u_{0}}, \quad W(\tau, \xi, \eta)=\frac{\bar{w}}{u_{0}}, \quad u_{c}=\frac{\bar{u}_{c}}{u_{0}}, \quad S=\frac{h}{D_{1}} \Sigma$.

Then the system (1)-(6) has parameters $\beta=\gamma \frac{D_{1}}{h^{2}}, \quad d=\frac{D_{2}}{D_{1}}, \quad K=\frac{u_{0}}{\varphi}, \quad P=\frac{D_{2}}{h \delta}$.

Let us make substitution $w=W-u_{c}$

$\frac{\partial u(\tau, \xi)}{\partial \tau}=\frac{\partial^{2} u(\tau, \xi)}{\partial \xi^{2}}+\left.P \frac{\partial w(\tau, \xi, \eta)}{\partial \eta}\right|_{\eta=0}-\beta u(\tau, \xi), \quad \xi>0, \tau>0 ;$

$u(0, \xi)=0, \quad u(\tau, 0)=1, \quad u(\tau, \infty)=0$

$\frac{\partial w(\tau, \xi, \eta)}{\partial \tau}=d \frac{\partial^{2} w(\tau, \xi, \eta)}{\partial \eta^{2}}, \quad t>0,0<y<\Sigma ;$

$w(0, \xi, \eta)=-u_{c}, \quad w(\tau, \xi, 0)=u(\tau, \xi)-u_{c}$,

$-\left.d \frac{\partial w}{\partial \eta}\right|_{\eta=S}=\left.H(w) w\right|_{\eta=S}$

$\frac{\partial S}{\partial \tau}=\left.K H(w) w\right|_{\eta=S}, \quad S(0, \xi)=0$.

Eqs. (7)-(11) are dimensionless problem of surface-reaction diffusion.

\section{Analytical solution}

In the course of experimental research [2,3] estimations for diffusion coefficients were obtained. Analysis showed that the estimation $D_{1} \gg D_{2}$ (or $d \ll 1$ ) is true for all substance pairs, whereas boundary movement inside the substrate occurs slowly (therefore $\dot{\Sigma}$ derivative can be considered negligible quantity, $\dot{\Sigma} \ll 1$ ). These estimations are actually necessary for surface-reaction diffusion mechanism realization. Then diffusion process characteristic time inside the substrate $t_{D}$ is much less than $t_{\Sigma}$ boundary motion time. Moreover, it is well known from the general theory of problems with mobile boundary [28] and appendixes $[19,23,29]$, that relaxation time is defined in the first place by front movement. Consequently, one need not to search the exact solution of Eq. (8) in the subtask (8)-(10), but approximately consider the $w_{\tau} \approx 0$ derivative as being negligible quantity. One can come to the same conclusion, using first approximation of differential series method [28], taking into account that $d \ll 1$. It should be noted that assumption $w_{\tau} \approx 0$ was confirmed by numerical simulation, therefore linear solution of inner problem (8)-(10) is well grounded:

$w(\tau, \xi, \eta)=\left(u(\tau, \xi)-u_{c}\right)\left(1-\frac{\eta}{d+S}\right)$,
$0 \leqslant \eta \leqslant S, \quad \xi: u(\tau, \xi)-u_{c}>0$
$w(\tau, \xi, 0)=u(\tau, \xi)-u_{c}, \quad \xi: u(\tau, \xi)-u_{c}<0$.

Let us insert this solution in problems (7)-(11) for functions $u(\tau, \xi)$ and $S(\tau, \xi)$ :

$\frac{\partial u(\tau, \xi)}{\partial \tau}=\frac{\partial^{2} u(\tau, \xi)}{\partial \xi^{2}}-F(\tau, \xi)-\beta u(\tau, \xi)$,

$u(\tau, 0)=1, \quad u(0, \xi)=0$

$\frac{\partial S}{\partial \tau}=K d \frac{\left(u-u_{c}\right)}{d+S} H\left(u-u_{c}\right), \quad S(0, \xi)=0 ;$

whereas $F(\tau, \xi)$ equals

$F(\tau, \xi)=P \frac{\left(u-u_{c}\right)}{d+S} H\left(u-u_{c}\right)$.

In writing system (13) and (14) the transition from $H(w)$ function to $H\left(u-u_{c}\right)$ is carried out on the basis of obtained expression (12) for $w(\tau, \xi, \eta)$. The replacement has no impact on the essence of the model, but simplifies the problem solution. 
Solution of the (13) and (14) system can be split up into three steps:

1. If diffusive outflow is absent $(F=0)$, then Eq. (14) has no sense, there is no penetration inside the substrate. Let us find diffusant concentration in the first approximation $u_{1}(\tau, \xi)$. One can construct solution through Laplace integral transformation.

2. The found first approximation $u_{1}(\tau, \xi)$ we apply to find reaction front in the first approximation $S_{1}(\tau, \xi)$. To this end we integrate Eq. (14), assuming that $u(\tau, \xi)=u_{1}(\tau, \xi)$.

3. Inserting the obtained first approximations $u_{1}(\tau, \xi)$ and $S(\tau, \xi)$ into function $F(\tau, \xi)$ and considering it as known heterogeneity of Eq. (13), once again solve (13). Thus we find $u(\tau, \xi)$ in the second approximation $u_{2}(\tau, \xi)$.

Let us proceed according to the plan. At first solve Eq. (13) when $F=0$ through Laplace integral transformation. Eq. (13) looks like that in images

$U_{1 \xi \xi}(s, \xi)-(s+\beta) U_{1}(s, \xi)=0, \quad U_{1}(s, \infty)=0, \quad U_{1}(s, 0)=1 / s$,

where $U_{1}(s, \xi)$ - image of Laplace transformation for the $u_{1}(\tau, \xi)$ function. The solution is the function

$U_{1}(s, \xi)=\frac{e^{-\xi \sqrt{\beta+s}}}{S}$.

Proceeding to preimages we get

$u_{1}(\tau, \xi)=\frac{1}{2}\left[e^{-\xi \sqrt{\beta}} \operatorname{erfc}\left(\frac{\xi}{2 \sqrt{\tau}}-\sqrt{\beta \tau}\right)+e^{\sqrt{\beta \xi}} \operatorname{erfc}\left(\frac{\xi}{2 \sqrt{\tau}}+\sqrt{\beta \tau}\right)\right]$.

Now we can integrate Eq. (14), considering that $u(\tau, \xi)=u_{1}(\tau, \xi)$

$S_{1}(\tau, \xi)=-d+\sqrt{d^{2}+2 K d \int_{0}^{\tau}\left(u_{1}-u_{c}\right) H\left(u-u_{c}\right) d \tau}$.

Proceed to the third step of calculations and find $u_{2}(\tau, \xi)$. Considering $F(\tau, \xi)$ to be known heterogeneity of Eq. (13), pass over to images of Laplace integral transformation

$U_{2 \xi \xi}(s, \xi)-(s+\beta) U_{2}(s, \xi)=\Phi(s, \xi)$,

$U_{2}(s, \infty)=0, \quad U_{2}(s, 0)=1 / s$,

where $U_{2}(s, \xi)$ - Laplace transformation image for the function $u_{2}(\tau, \xi) ; \Phi(s, \xi)$ - transformation image for the function $F(\tau, \xi)$.

The general solution of heterogeneous equation looks like

$$
\begin{aligned}
U_{2}(s, \xi)= & -\int_{0}^{\xi} \frac{\Phi e^{-(\xi-y) \sqrt{\beta+s}}}{2 \sqrt{\beta+s}} d y-\int_{\xi}^{\infty} \frac{\Phi e^{-(y-\xi) \sqrt{\beta+s}}}{2 \sqrt{\beta+s}} d y+D_{1} e^{-\xi \sqrt{\beta+s}} \\
& +D_{2} e^{\xi \sqrt{\beta+s}} .
\end{aligned}
$$

Constants are found through boundary condition:

$D_{1}=\frac{1}{s}+\int_{0}^{\infty} \frac{\Phi e^{-y \sqrt{\beta+s}}}{2 \sqrt{\beta+s}} d y, \quad D_{2}=0$.

Finally the boundary problem (18) looks like

$$
\begin{aligned}
U_{2}(s, \xi)= & \frac{1}{s} e^{-\xi \sqrt{\beta+s}}+\int_{0}^{\infty} \frac{\Phi e^{-(\xi+y)} \sqrt{\beta+s}}{2 \sqrt{\beta+s}} d y-\int_{0}^{\xi} \frac{\Phi e^{-(\xi-y) \sqrt{\beta+s}}}{2 \sqrt{\beta+s}} d y \\
& -\int_{\xi}^{\infty} \frac{\Phi e^{(\xi-y) \sqrt{\beta+s}}}{2 \sqrt{\beta+s}} d y .
\end{aligned}
$$

Proceeding back to preimages, using convolution theorem for Laplace transform and Eq. (16), we get the solution of the initial problem (13) in second approximation

$$
\begin{aligned}
u_{2}(\tau, \xi)= & \frac{1}{2} \int_{0}^{\infty} d y \int_{0}^{\tau} d t\left\{\frac{F(t, \xi) e^{-\frac{(\xi+y)^{2}}{4(\tau-t)}-\beta(\tau-t)}}{\sqrt{\pi(\tau-t)}}\right\} \\
& -\frac{1}{2} \int_{0}^{\infty} d y \int_{0}^{\tau} d t\left\{\frac{F(t, \xi) e^{-\frac{(\xi-y)^{2}}{4(\tau-t)}-\beta(\tau-t)}}{\sqrt{\pi(\tau-t)}}\right\}+u_{1}(\tau, \xi),
\end{aligned}
$$

where

$$
F(\tau, \xi)=P \frac{\left(u_{1}-u_{c}\right) H\left(u_{1}-u_{c}\right)}{\sqrt{d^{2}+2 K d \int_{0}^{\tau}\left(u_{1}-u_{c}\right) H\left(u-u_{c}\right) d \tau}} .
$$

\section{Numerical solution}

One should note the system (7)-(11) properties since they are important for numerical solution. Firstly, it is defined on an unbounded domain. Secondly, Eqs. (8)-(11) are a parabolic type problem with mobile unknown boundary. Even though there are known methods for solving equations with mentioned peculiarities in this problem they are combined in one system and should be solved simultaneously.

In order to use traditional finite-difference schemes we should map the changing area $[0, \infty) \times[0, S(\tau, \xi)]$ to the square $[0,1] \times[0,1]$ through transformation of coordinates: $\bar{x}=1 /(\xi+1)$ and $\eta=\bar{y} S(\tau, \xi)$.

After the change the problem is solved iteratively in two steps. Firstly, one should find $u_{k i}=u\left(k \tau, i h_{1}\right)$ from the equation

$$
\begin{aligned}
& \frac{u_{k i}^{(r)}-u_{k-1, i}}{\lambda}= \bar{x}_{i}^{4} \frac{\left(u_{k, i+1}^{(r)}-2 u_{k, i}^{(s)}+u_{k, i-1}^{(r)}\right)}{h_{1}^{2}}+\bar{x}_{i}^{3} \frac{\left(u_{k, i+1}^{(r)}-u_{k, i-1}^{(r)}\right)}{h_{1}} \\
&+\frac{P}{S_{k i}^{(r-1)}} \frac{\left(w_{k i, 2}^{(r-1)}-w_{k i, 0}^{(r-1)}\right)}{2 h_{2}}-\beta u_{k i}, \\
& i=1 . . N-1 \quad u_{0 i}^{(r)}=0, u_{k 0}^{(r)}=0, u_{k N}^{(r)}=1, i=0, \ldots, N,
\end{aligned}
$$

where $N=1 / h_{1}, w_{k i j}=w\left(k \lambda, i h_{1}, j h_{2}\right)$. Concerning the unknown $\left\{u_{k i}^{(r)}\right\}_{i=0 . N}$ the system (20) looks like linear system with tridiagonal matrix, that is why its solution is easily found using tridiagonal matrix algorithm (Thomas algorithm). The value of the previous step on time coordinate is taken as an initial value. The iterations go on unless the values on iterations are similar.

Secondly, nonlinear inner problem for $w$ is solved. If $v_{j}=w_{k i j}$ for $j=1 . . M$, then vector of the unknown will be $\mathbf{v}=\left(v_{0}, v_{1}, \ldots, v_{M}\right)^{t}$. Let us also consider vector function $\Psi(\mathbf{v})$, thanks to which one can write down the inner problem (8)-(10) like $\boldsymbol{\Psi}\left(v_{0}, v_{1}, \ldots, v_{M}\right)=0$, with this the function $\Psi(\mathbf{v})$ itself is defined in the following way

$\Psi_{0}(\mathbf{v})=v_{0}-u_{k i}-u_{c}$

$$
\begin{aligned}
\Psi_{j}(\mathbf{v})= & \left(v_{j}-w_{k-1, i j}\right) S_{k i}^{2}-\frac{d \lambda}{h_{2}^{2}}\left(v_{j+1}-2 v_{j}+v_{j-1}\right) \\
& -\frac{\bar{y}_{j} \lambda}{2 h_{2}} K \mathrm{H}\left(v_{j}\right) S_{k i} v_{M}\left(v_{j+1}-v_{j-1}\right), j=\overline{1, M-1} \\
\Psi_{M}(\mathbf{v})= & \mathrm{H}\left(v_{j}\right) S_{k i} v_{M}+d \frac{v_{M}-v_{M-1}}{h_{2}} .
\end{aligned}
$$

Now to find $w$ (for each $r$ iteration of outer problem) one should solve the system of nonlinear equations $\boldsymbol{\Psi}\left(v_{0}, v_{1}, \ldots, v_{M}\right)=0$ through Newton's method:

$$
v_{j}^{(q+1)}=v_{j}^{(q)}+J^{-1}\left(\mathbf{v}^{(q)}\right) \boldsymbol{\Psi}\left(\mathbf{v}^{(q)}\right),
$$

where $J(\mathbf{v})$ - Jacobian matrix, $J(\mathbf{v})=\left(\partial \Psi_{i} / \partial v_{j}\right)$. The calculations are carried out unless the corrective increments $\Delta \mathbf{v}=v_{j}^{(q+1)}-v_{j}^{(q)}$ are sufficiently small according to Euclidean norm. 
Jacobian matrix $J(\mathbf{v})$ for internal problem has the structure of tridiagonal matrix with filled last column, which makes possible finding the $\Delta \mathbf{v}$ value from the system $J\left(\mathbf{v}^{(q)}\right) \Delta \mathbf{v}=\boldsymbol{\Psi}\left(\mathbf{v}^{(q)}\right)$ through modified tridiagonal matrix algorithm.

Values from the previous time layer were taken as initial conditions both for outer and inner problem and Newton's method (21) quite rapidly came to solution. Numerical algorithm realization was carried out through $\mathrm{C}++$.

\section{Result and discussion}

Since reaction and front movement inside the substrate occur only on condition that $w(\tau, \xi, \eta)>0$, and according to expression (12) $w(\tau, \xi, \eta)>0$ if and only if $\left(u-u_{c}\right)>0$, then movement of the reacted surface layer is directly connected to concentration level movement $u(\tau, \xi)=u_{c}$. This movement was found from analytical solution (19) thanks to computer algebra system MathCad. Fig. 3 shows calculation results if $P=0, P=0.004, P=0.008$.

The curves 1 and 4 show concentration level movement in the first approximation $u_{1}(\tau, \xi)=u_{c}$ according to Eq. (16) without diffusive outflow from the substrate $(P=0)$. One can see that a single assumption concerning evaporation is sufficient enough for stabilization of distribution $u_{1}(\tau, \xi)$. The process intensity has no importance. In any case distribution becomes stationary. The rest of the curves $(2,3,5,6)$ illustrate the diffusive outflow influence. The outflow decelerates reaching the stationary distribution, which can be regarded as ultimate. One should also note that even small variation in parameter values $(P=0.004, P=0.008)$ has a serious impact on "convergence" to stationary condition.

It is interesting to compare analytical (19) and numerical solutions. The curves 2 and 3 (similarly to 5 and 6 ) interflow in the course of time and simultaneously slowly reach the ultimate level when $P=0$. In this case both solutions verify and validate each other: convergence of numerical algorithm is qualitatively confirmed as well as correctness of the second approximation $u_{2}(\tau, \xi)$ (19) construction.

Let us explain the difference between numerical and analytical solutions in the initial time point. At the first stage it was considered that diffusion outflow inside the substrate is absent $(P=0)$ and the whole substance is distributed over the surface. This means that the solution $u_{1}(\tau, \xi)$ is too overrated and actually there is much less diffusing substance on the surface than $u_{1}(\tau, \xi)$. Then, based on the $u_{1}(\tau, \xi)$, the second approximation for the front $S_{1}(\tau, \xi)$ was constructed. This approximation shows that the front is bedded deeper than it really is. At the third stage considering $F(\tau, \xi)$ to be known heterogeneity of Eq. (13), the second approximation $u_{2}(\tau, \xi)$ was constructed. Since for constructing the $F(\tau, \xi)$ overrated

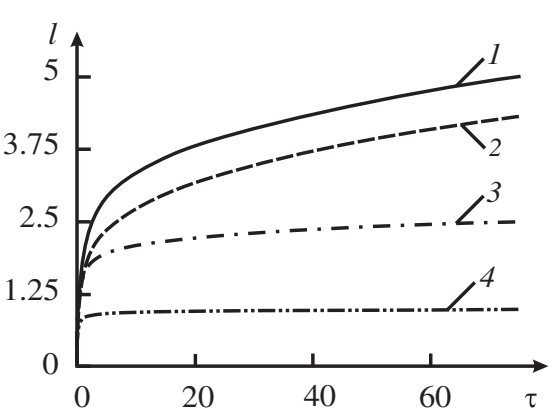

Fig. 4. The curve 1 - model without reaction reversibility and without evaporation; the curve 2 - model with evaporation; the curve 3 - model with reaction reversibility; the curve 4 - generalized model of surface-reaction diffusion. The models' parameters: $P=1, \beta=0.2, u_{c}=0.01$.

values $u_{1}(\tau, \xi)$ and $S_{1}(\tau, \xi)$ have been used, then the $F(\tau, \xi)$ function expresses excessive outflow from the surface. Therefore the obtained second approximation $u_{2}(\tau, \xi)$ illustrates underrated concentration distribution $u(\tau, \xi)$. As a result the solution of $u_{2}(\tau, \xi)$ gives lower estimate and consequently the constructed movement of surface layer length is also a low estimate. In the course of time the differences in solutions disappear. All the above is illustrated in Fig. 3 by the curves 2 and 3 (similarly to 5 and 6 ).

Let us now compare four models of surface-reaction diffusion. Fig. 4 shows results of numerical algorithm work for 4 models: the 1 curve-is a model without reaction reversibility and without evaporation $\left(\beta=0, u_{c}=0\right)$; the 2 curve-is a model with evaporation $\left(\beta \neq 0, u_{c}=0\right)$; the 3 curve-is a model with reaction reversibility $\left(\beta=0, u_{c} \neq 0\right)$; the 4 curve-is a generalized model of surface-reaction diffusion.

Let us analyze each model.

1. If there is no evaporation and reaction reversibility then the principal influence on the surface layer $l(\tau)$ movement has diffusion fluxes redistribution (curve 1, Fig. 4). In this case the border $l(\tau)$ is defined from the equality $S(\tau, l(\tau)=\varepsilon)$, where $\varepsilon \ll 1$. It was obtained that

$$
\begin{aligned}
l(\tau)= & \sqrt{\frac{3}{P}} \sqrt{3 d+2 S_{0}}+\sqrt{\frac{d}{P}} \ln \left(\frac{\sqrt{3 d+2 S_{0}}-\sqrt{3 d}}{\sqrt{3 d+2 S_{0}}+\sqrt{3 d}}\right)-\sqrt{\frac{3}{P}} \\
& \times \sqrt{3 d+2 \varepsilon}-\sqrt{\frac{d}{P}} \ln \left(\frac{\sqrt{3 d+2 \varepsilon}-\sqrt{3 d}}{\sqrt{3 d+2 \varepsilon}+\sqrt{3 d}}\right),
\end{aligned}
$$

where $S_{0}=S(\tau, 0)=d(\sqrt{1+2 K \tau / d}-1)$. It turns out that surface layer length is connected with time $l \sim \tau^{1 / 4}$. Thus the classic
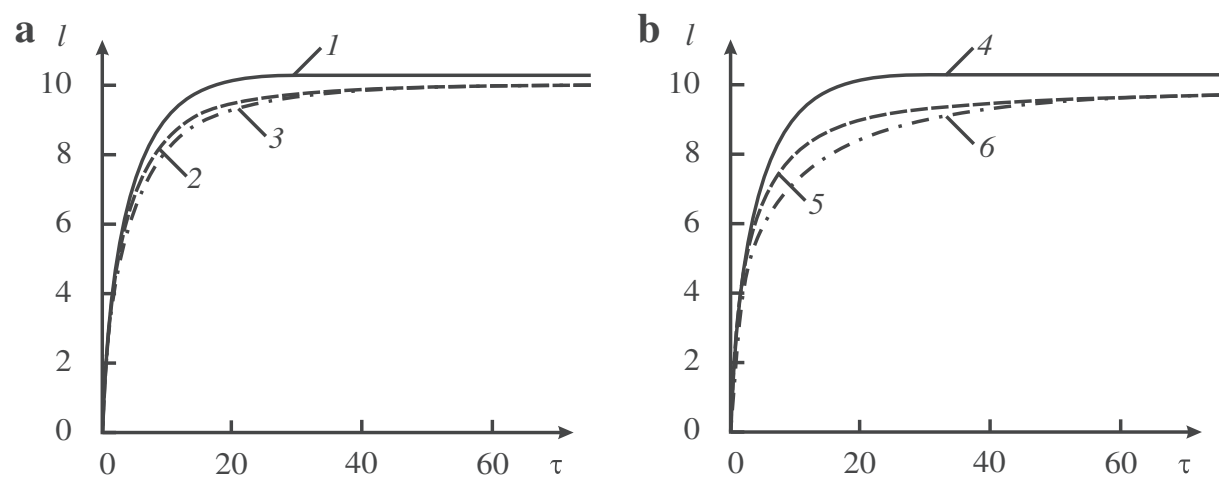

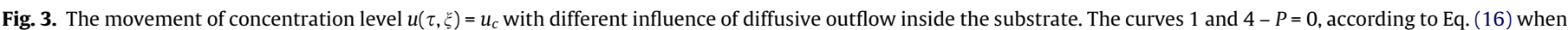

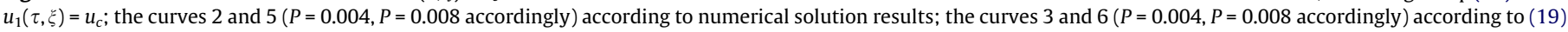
when $u_{2}(\tau, \xi)=u_{c}$. 
Fischer's result of diffusive penetration estimation (diffusion model with outflow [8]) is confirmed. Certainly one cannot speak of surface layer stop.

2. During the research into model with diffusant evaporation (curve 2, Fig. 4) the logarithmic law $l \sim \ln (t)$ of reaction front distribution over the substrate surface is obtained

$$
l(\tau)=\frac{1}{\sqrt{2}}\left[I\left(S_{0}\right)-I(\varepsilon)\right]
$$

where $S_{0}(\tau)$ is the defined above function, and $I(v)$ is the function of arbitrary argument $v$ looks like

$$
\begin{array}{r}
I(v)=\frac{1}{\sqrt{a}} \ln \left(\frac{b}{2 \sqrt{a}}+v \sqrt{a}+\sqrt{a v^{2}+b v+c}\right) \\
-\frac{d}{\sqrt{c}} \ln \left(\frac{b}{2}+\frac{c}{v}+\frac{\sqrt{c}}{v} \sqrt{a v^{2}+b v+c}\right), \\
a=\frac{\beta}{8}, \quad b=\frac{P}{3}+\frac{\beta d}{2}, \quad c=\frac{P d}{2}+\frac{\beta d^{2}}{2} .
\end{array}
$$

The thing is that diffusant concentration on the surface in the course of time reaches stationary value (for instance see first approximation $u_{1}(\tau, \xi)$ ). However this stationary is not followed by reaction front stop, since diffusant penetration inside the substrate does not cease and according to assumption 2 of the problem statement the reaction occurs inside the substrate.

3. The model with reaction reversibility (curve 3, Fig. 4) does not make it possible to construct an analytical solution and estimate front distribution rate. However according to the view of the curve one can see that this rate falls down. Assumption concerning reaction reversibility has an essential influence but not critical.

4. The generalized model with reaction reversibility and diffusant evaporation (curve 4, Fig. 4) shows the most plausible law of reaction front movement. The evaporation provides stationary diffusant concentration distribution over the surface in the course of time. Moreover, reaction interaction threshold $u_{c}$ guarantees that front movement will not occur in the areas, where $u(\tau, \xi)<u_{c}$. In other words stationary diffusant concentration distribution over the surface rigidly limits reaction front distribution.

Thus the principal possibility of stop (sharp deceleration) is conceivable and everything depends on model parameters $u_{c}, P$ and $\beta$ correlation. If the outflow is considerable $(P \gg \beta)$, then stationary diffusant concentration distribution over the surface is fixed slowly, and effect of reaction front deceleration over the sur-

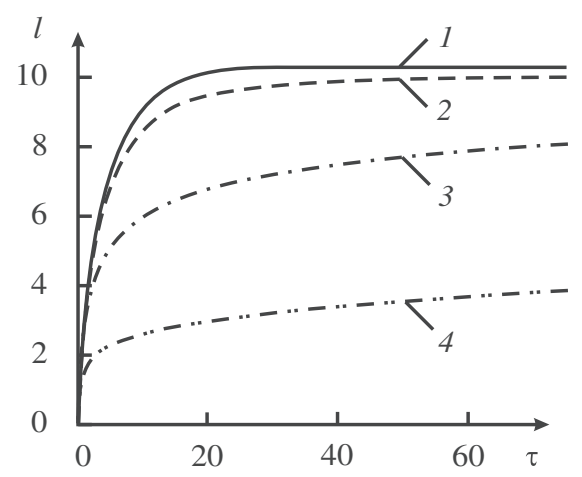

Fig. 5. Surface layer length with different parameter $P$ values. Curve $1-P=0$; curve $2-P=0.004$; curve $3-P=0.04$; curve $4-P=0.4$. Other parameters of the model $\beta=0.2, u_{c}=0.01$.

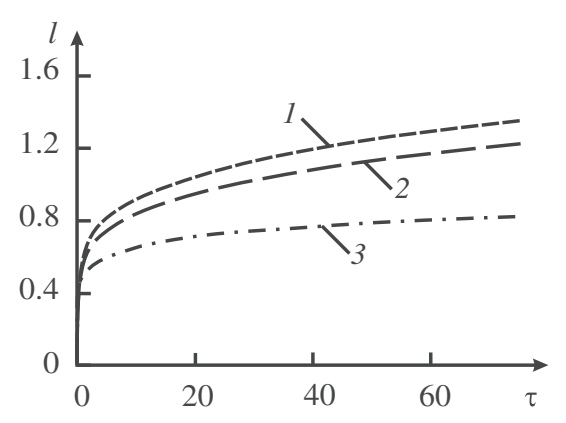

Fig. 6. Surface layer length with different parameter $\beta$ values. Curve $1-\beta=0.002$, curve $2-\beta=0.02$, curve $3-\beta=0.2$. Other parameters of the model $P=0.4, u_{c}=0.01$.

face during the time of numerical experiment is virtually not observed (curve 4, Fig. 5 and curve 1, Fig. 6). In this case the model is similar to the model without evaporation, but with reaction reversibility (model 3 ). Otherwise $(P \sim \beta)$ the evaporation occurs rapidly and imposes stationary concentration distribution, and when $u_{c} \neq 0$ the sharp deceleration effect is observed (curve 2, Fig. 5 and curve 3, Fig. 6 ). The curve 4 (Fig. 4 ) corresponds to the fact that at $D_{1}=10^{-3}, D_{2}=10^{-6}, h=5 \cdot 10^{-4}, \gamma=5 \cdot 10^{-4}, \delta=10^{-3}$ the length of the surface layer $l_{s}$ reaches a level of about $2 \mathrm{~mm}$ and practically does not change in the course of time. This behavior is similar to that observed in Fig. 2. The above values are in good agreement with estimate of parameters. The more $u_{c}$, the less limit value of the surface layer length $l_{s}$. One should note that with presence of inexhaustible source (in all models) the quantity of evaporated substance is not essential for the issue; the sufficient diffusant penetration inside the substrate will be provided by the percentage of general diffusive flux, inflowing on the surface.

In conclusion let us emphasize that we constructed the mathematical model surface-reaction diffusion in which we take into account the impact of the reaction reversibility, evaporation, redistribution of flows and does not take into account the porous structure of the substrate. Nevertheless, model predicts stabilization of the surface layer length $l_{s}$. Analytical and numerical solution methods, which we used in this article, can be useful to solve similar Stefan-like problem.

\section{Acknowledgements}

We are grateful for partial support from the Federal Target Program "Scientific and Scientific-pedagogical Personnel of Innovative Russia" in 2009-2013, the Russian Foundation for Basic Research (Project No. 10-01-96045 Ural) and Ural Federal University.

\section{References}

[1] T.A. Langhoff, E. Schnack, Modelling chemical vapour infiltration of pyrolytic carbon as moving boundary problem, Chem. Eng. Sci. 63 (2008) 3948-3959.

[2] A. Neiman, A. Guseva, M. Trifonova, Surface reaction diffusion during formation of molybdates and tungstates, Solid State Ionics 41 (142) (2001) 321-329.

[3] A. Guseva, A. Neiman, M. Trifonova, E. Konisheva, E. Gorbunova, The interface transport of $\mathrm{V}_{2} \mathrm{O}_{5}$ and $\mathrm{WO}_{3}$ into $\mathrm{CaMo}(\mathrm{W}) \mathrm{O}_{4}$ stimulated by an electric field, Surf. Sci. 507 (510) (2002) 140-145.

[4] J.C. Fisher, Calculation of diffusion penetration curves for surface and grain boundary diffusion, J. Appl. Phys. 22 (1951) 74-77.

[5] T. Suzuoka, Lattice and grain boundary diffusion in polycrystals, Trans. Jpn. Inst. Met. 2 (1961) 25-33.

[6] Yu. M. Mishin, Defect Diffus. Forum 194-199 (2001) 1113-1126.

[7] B.S. Bokshteyn, Ch.V. Kopetskiy, L.S. Shvindlerman, Thermodynamics and Kinetics of Grain Boundaies in Metal, Nauka Publishing House, Moscow, 1986 (in Russian).

[8] K. Chuntonov, J. Setina, New lithium gas sorbents. I. The evaporable variant, J. Alloy Compd. 455 (2008) 489-496. 
[9] K. Chuntonov, A. Ivanov, D. Permikin, New lithium gas sorbents. II. A mathematical model of the evaporation process, J. Alloy Compd. 456 (2008) 187-193.

[10] K. Chuntonov, J. Setina, A. Ivanov, D. Permikin, New lithium gas sorbents. III. Experimental data on evaporation, J. Alloy Compd. 460 (2008) 357-362.

[11] K. Chuntonov, A. Ivanov, D. Permikin, New lithium gas sorbents. IV. Application to MEMS devices, J. Alloy Compd. 471 (2009) 211-216.

[12] K. Hishida, K. Nishiyama, On the variation of heat exchange and evaporation at the sea surface in the Western North Pacific Ocean, J. Oceanogr. Soc. Jpn. 25 (1) (1969) 1-9.

[13] L. Bengtsson, Evaporation from a snow cover, Nordic Hydrol. 11 (1980) 221 234

[14] A.L. AL-Khlaifat, Dead sea rate of evaporation, Am. J. Appl. Sci. 5 (8) (2008) 934-942.

[15] LingQian Chang, ChunXiu Liu, YinZeng He, HongHui Xiao, XinXia Cai, Smallvolume solution current-time behavior study for application in reverse iontophoresis-based non-invasive blood glucose monitoring, Sci. China Chem. 54 (1) (2011) 223-230.

[16] N.A. Fuchs, Evaporation and Droplet Growth in Gaseous Media, Pergamon, NY, 1959.

[17] B.B. Hicks, Deposition of atmospheric acidity, in: M. Radojevic, R.M. Harrison (Eds.), Atmospheric Acidity: Sources, Consequences and Abatement, Springer, 2007, pp. 178-180.

[18] B.S. Mazumder, Suvadip Paul, Dispersion of reactive species with reversible and irreversible wall reactions, Heat Mass Transfer (2011) 1-12.

[19] D.V. Alexandrov, Self-similar solidification: morphological stability of the regime, Int. J. Heat Mass Transfer 47 (2004) 1383-1389.
[20] F.J. Vermolena, E. Javierre, On the construction of analytic solutions for a diffusion reaction equation with a discontinuous switch mechanism, J. Comput. Appl. Math. 231 (2009) 983-1003.

[21] A. Muntean, M. Böhm, Moving-boundary problem for concrete carbonation: global existence and uniqueness of weak solutions, J. Math. Anal. Appl. 350 (2009) 234-251.

[22] A. Muntean, M. Böhm, J. Kropp, Moving carbonation fronts in concrete: a moving-sharp-interface approach, Chem. Eng. Sci. 66 (2011) 538-547.

[23] D.V. Alexandrov, A.P. Malygin, The Stefan problem for unsteady-state evaporation of a volatile component in the solid-liquid-gas systems: exact analytical solution, Int. J. Heat Mass Transfer 53 (2010) 2790-2794.

[24] C. Vuik, Some historical notes on the Stefan problem, Nieuw Archief voorWiskunde 4e Serie (11) (1993) 157-167.

[25] B. Sarler, Stefan's work on solid-liquid phase changes, Eng. Anal. Bound. Elem. 16 (1995) 83-92.

[26] J.S. Wettlaufer, The Stefan problem: polar exploration and the mathematics of moving boundaries, Festschrift 150 Jahre Institut für Met und Geophysik, Univ. Wien, Styria, Graz, 2001.

[27] J. Crepeau, Josef Stefan and his contributions to heat transfer, in: Proc. ASME 2008 Heat Transfer Conf., HT2008, Jacksonville, Florida, USA, Paper 56073, 8p., August 10-14, 2008.

[28] E.M. Kartashov, G.S. Krotov, Analytical solution of the single-phase Stefan problem, Math. Models Comput. Simul. (2009) 180-188.

[29] Suvadip Paul, B.S. Mazumder, Effects of nonlinear chemical reactions on the transport coefficients associated with steady and oscillatory flows through a tube, Int. J. Heat Mass Transfer 54 (2011) 75-85. 